known to increase the risk of serious skin reactions (see Ped Neurol Briefs May $1992 ; \underline{5}: 37)$.

\title{
SEIZURES AND CEREBRAL CYSTICERCOSIS
}

The results of a long-term retrospective study of 240 patients with seizures and cysticercosis of the brain parenchyma are reported from the Instituto Nacional de Neurologio y Neurocirugia Manuel Velasco Suarez de Mexico, Mexico City. Of 118 patients who received cysticidal therapy (albendazole, prasiquantel, or both) for lesions without inflammation on imaging studies, there was an $82 \%$ reduction in the mean number of brain cysts and a $95 \%$ reduction in the mean frequency of seizures. After 3 years of follow-up, $54 \%$ of patients were seizure free. By contrast, the untreated patients averaged 11 seizures per year and none was seizure free. Of 58 patients with inflammation around cysts who did not receive cysticidal medication, there was a $74 \%$ reduction in the frequency of seizures and $31 \%$ became seizure free. After surgical treatment of unmedicated patients there was an $87 \%$ reduction in seizure frequency and $40 \%$ became seizure free. (Vazquez V, Sotelo J. The course of seizures after treatment for cerebral cysticercosis. N Engl J Med Sept 3, 1992; 327:696-701.) (Reprints: Dr. Sotelo, National Institue of Neurology and Neurosurgery, Insurgentes sur 3877, 14269, Mexico City, Mexico.)

COMMENT. The outcome for patients with epilepsy due to neurocysticercosis was better after treatment with cysticidal agents than when the primary disease was left to follow its natural course. There were fewer seizures after medical treatment than after surgical excision of the cyst. In the patients with inflammation around cysts, spontaneous resolution of the cysts occurred frequently but the number of seizures per year in this group was $31 \%$ higher than in those who received medical treatment with cysticidal drugs. Granulomas remaining after the cysts destruction may account for the high incidence of persistent seizures. The patients in this study were adults, but most had suffered from frequent seizures for many years because of persistence of the paracystic epileptic focus.

Children in endemic areas who have seizures related to cysticercosis should be considered for cysticidal therapy at the time of diagnosis. The use of these drugs in children may require further definition.

Cysticercosis of the CNS is rare in the United States, but accounts for $25 \%$ of intracranial tumors in Mexico. Increased intracranial pressure is found in about $75 \%$ of cases (Menkes JH. Textbook of Child Neurology $3 \mathrm{rd}$ Ed. Philadelphia. Lee and Febiger 1981). 